\title{
Surface diffusion coefficients for room acoustics: Free-field measures
}

\author{
Tristan J. Hargreaves, Trevor J. Cox, ${ }^{\text {a) }}$ and Y. W. Lam \\ School of Acoustics and Electronic Engineering, University of Salford, Salford M5 4WT, United Kingdom \\ Peter D'Antonio \\ RPG Diffusor Systems, Inc., 651-c Commerce Drive, Upper Marlboro, Maryland 20772
}

(Received 14 October 1999; revised 10 July 2000; accepted 15 July 2000)

\begin{abstract}
A surface diffusion coefficient is needed in room acoustics to enable the quality of diffusing surfaces to be evaluated. It may also facilitate more accurate geometric room acoustic models. This paper concentrates on diffusion coefficients derived from free-field polar responses. An extensive set of two- and three-dimensional measurements and predictions was used to test the worth of different diffusion coefficient definitions. The merits and problems associated with these types of coefficients are discussed, and past parameters reviewed. Two new coefficients are described. The new measure based on the autocorrelation function is forwarded as the best free-field coefficient. The strengths and weaknesses of the coefficient are defined. (C) 2000 Acoustical Society of America.
\end{abstract}

[S0001-4966(00)04810-4]

PACS numbers: 43.55.Br, 43.20.El [JDQ]

\section{INTRODUCTION}

Surface diffusion can play a key role in determining the sound field within an enclosed space. For example, the correct use of diffusion in performance spaces may enhance the acoustic for both the audience and musicians. ${ }^{1}$ Given the important role that diffuse reflections can play in determining the sound field, it is necessary to have a measure to gauge the degree of diffusion created by a surface. Only by having a numerical measure of surface diffusion is it possible to readily compare the performance of different treatments, and to develop design specifications for diffusers. Indeed, by creating a language to describe the degree of diffusion, it is hoped to improve the understanding of diffuse reflection phenomena among practitioners.

The development of a diffusion coefficient is also of interest to developers of geometric room acoustic models. A round robin test of geometric room acoustic computer models $^{2}$ showed that the common feature of the most successful predictions was the inclusion of some form of diffusion modeling. The implementation of diffusion in geometric models currently requires some single-figure random incidence measure- a diffusion coefficient. Unfortunately, at the moment the diffusion coefficient has to be chosen empirically ${ }^{3}$ since there are no clear relationships between the physical properties of a surface and the diffusion coefficients adopted by computer models. Indeed, $\mathrm{Lam}^{4}$ showed that existing diffusion modeling algorithms generally require different diffusion coefficient values to model the same room surface.

The current interest in diffusion coefficients is acknowledged by the fact that two international standard working groups are currently looking at this issue. The diffusion coefficient outlined in this paper is the method likely to be enshrined in one of the international standards.

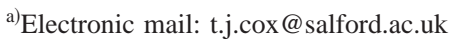

The literature contains a number of methods that have been proposed to characterize the diffuse scattering from surfaces. Unfortunately, there is not one diffusion coefficient in the literature or proposed in this paper that does not have flaws. While at first it may appear possible to produce a watertight definition of a diffusion coefficient, detailed examination reveals that it is impossible. The choice of diffusion coefficient is application dependent. While this may appear unsatisfactory, it should be remembered that room acoustics has been using an absorption coefficient for a hundred years, which has well-defined limitations in application. For example, there are two primary techniques for measuring absorption: the impedance tube and reverberation chamber methods. Each method has advantages and disadvantages and is used for different reasons.

In an analogous manner, the methods for characterizing diffusion can be classified either as free- or diffuse field. The diffuse-field methods have the advantage of quickly obtaining a random incidence coefficient, but are difficult to predict. The free-field methods are often more laborious to carry out, but can be readily predicted. Probably the best known diffuse-field method is the technique suggested by Mommertz and Vorländer. ${ }^{5,6}$ This method looks at the invariant and variant portions of the sound-pressure decay in a reverberation chamber as the test surface is rotated. Another diffuse field method $^{7}$ investigates the effect that surface diffusers have on the diffuseness of the space.

This paper, however, concentrates on free-field methods. Most publications have dealt with diffusion coefficients based on polar distributions. Essentially, these coefficients gauge the spatial evenness of energy scattering around the surface, in a way similar to how the omnidirectionality of a sound source might be tested. A different type of free-field method investigates how much energy is scattered into and away from the specular reflection angle. This latter method appears to grow out of the type of definition certain geometric computer models have used in diffusion modeling. In this 
case complete diffusion is defined as the case where no energy is reflected into the specular reflection direction. The free-field method of Mommertz and Vorländer ${ }^{5}$ can be used to measure this type of coefficient. In a similar way to the diffuse-field technique, the measurement method gauges the invariance of the scattered pressure to surface movements measured at the specular reflection position. The diffusion coefficient derived is then the ratio of nonspecular to total reflected energy.

In this paper, however, the concern will be with the ability of diffusers to uniformly scatter in all directions, rather than with just the ability of a surface to move energy away from the specular angles. This restriction is placed because of experiences of diffuser design that some of the authors have. From the standpoint of the diffuser designer, it is important that a diffusion coefficient differentiates between redirection and dispersion. Diffusers are usually applied to treat first-order reflections, for example to prevent echoes from the rear wall of concert halls. If all the diffuser achieves is redirection, there is a risk that the echo problem will simply move to another place in the hall. On the other hand, if the diffuser achieves dispersion, this has the potential to reduce the echo problem without creating new difficulties for other listeners. For this reason, despite all its merits, the Mommertz and Vorländer free-field method will not be discussed further here.

Consequently, this paper returns to its main concern, free-field methods based on polar distributions. The general method is as follows. First, the scattering from a surface is measured or predicted in terms of a polar distribution. Then, the diffusion coefficient is a frequency-dependent, single figure of merit derived from the polar distribution. This is evaluated in one-third octaves, which has the advantage of smoothing out some of the local variations in the polar responses, so the diffusion coefficient is based more on the overall envelope. There have been various statistical operations suggested to calculate a diffusion coefficient from the polar distributions: standard deviation ${ }^{8-10}$ directivity, ${ }^{11,12}$ specular zone, and spherical harmonics. ${ }^{13}$ In any such data reduction, there is a risk of losing essential detail. Diffusion coefficients, however, have been applied to enable the quality of specialist diffusing surfaces to be evaluated and designed, for example by using the diffusion coefficient as a cost function in a numerical optimization scheme. ${ }^{8,9}$ Consequently, this supports the common belief that a single figure of merit can be useful. The main aim of this paper is to review the previously published diffusion coefficients based on polar distributions, and to forward a new coefficient based on the autocorrelation coefficient which seems to offer significant advantages over previous published techniques.

\section{EVALUATION CRITERIA}

The diffusion coefficients must be evaluated against a set of criteria. For the basis of this project, it was decided that an ideal diffusion coefficient would:

(i) have a solid physical basis;

(ii) be clear in definition and concept, and related to the current role of diffusion in room acoustics;

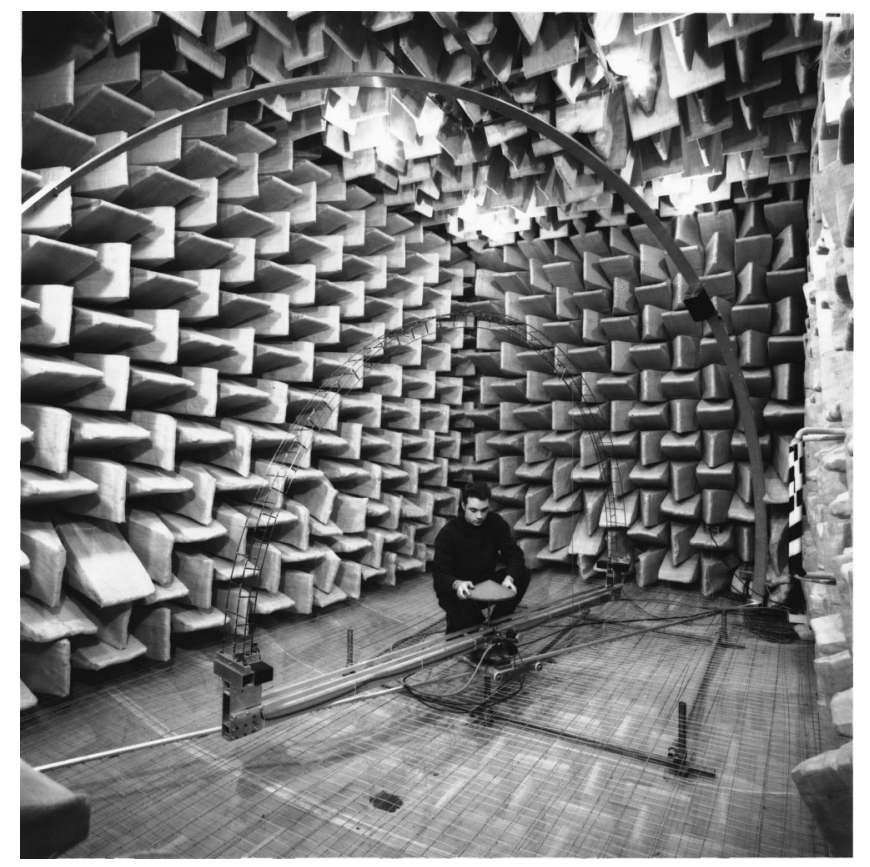

FIG. 1. Photograph of the automated goniometer for measuring hemispherical scattering being assembled in an anechoic chamber at the University of Salford. The lower part of the structure is covered with absorbent when operating.

(iii) consistently evaluate and rank the performance of diffusers;

(iv) apply to all the different surfaces and geometries found in rooms;

(v) be measurable by a simple process;

(vi) be bounded;

(vii) be easy to predict.

The various diffusion definitions found in literature, and new ones developed during the project were tested against the above criteria to test their suitability. This could be done by combining philosophical thought experiments with measurements and predictions of the coefficients for a wide variety of surfaces. It was also important to relate the assessment to current industry practice in diffuser design, and the application of diffusion in geometric room acoustic models.

\section{MEASUREMENT AND PREDICTION}

There are various techniques for obtaining the scattered pressure distributions to enable the coefficients to be calculated. Boundary element methods (BEMs) have been shown to be accurate in predicting the scattering from a variety of diffusing surfaces both in two and three dimensions ${ }^{14-16}$ and so have been used for this project. Measurements on reflecting surfaces were based on maximum length sequence signals using time gating to separate the reflected from the incident sound. Such a system has been used in the past to enable measurements to be made in a single plane on a semicircle. ${ }^{17} \mathrm{~A}$ capability to measure the surface scattering over the hemisphere using a goniometer was especially developed for this project. This is shown in Fig. 1. Measure- 

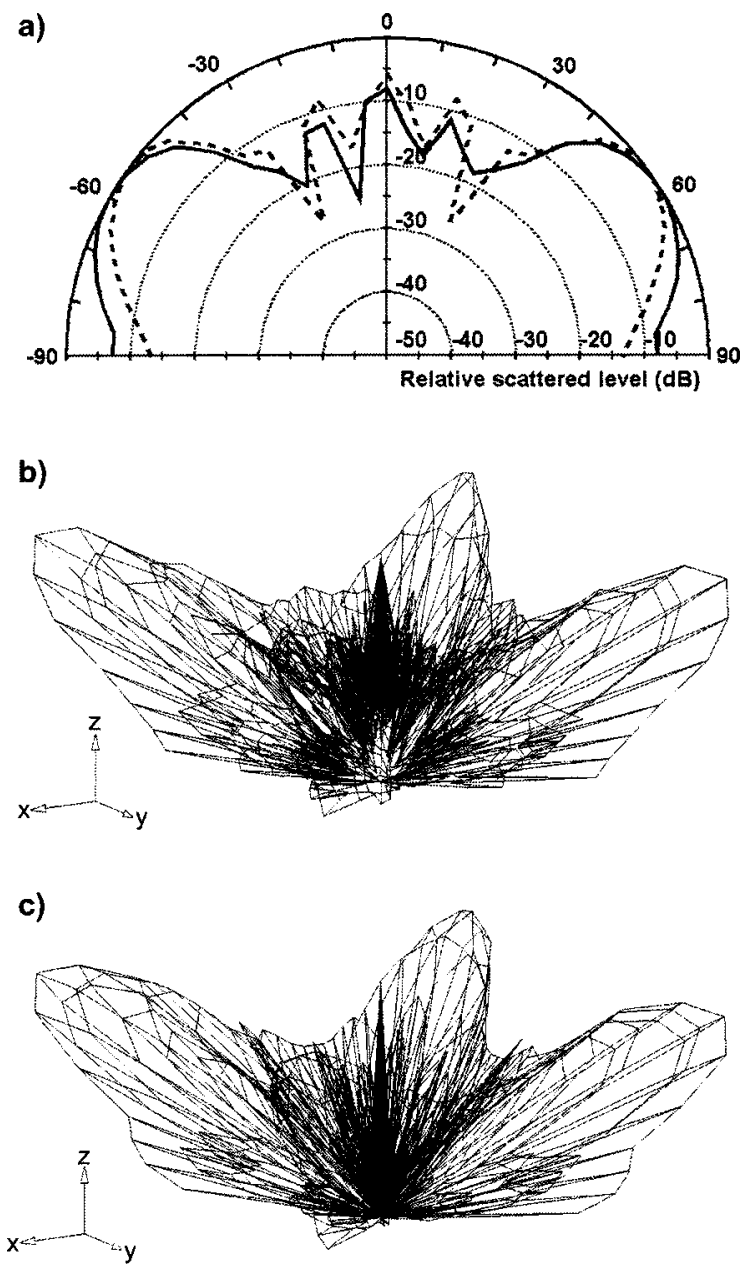

FIG. 2. Comparison of measured and predicted polar responses. (a) Two-dimensional measurement, --- single-plane BEM prediction for square-based pyramid, $1 \mathrm{kHz}$, normal incidence source. (b) Threedimensional measurement for square-based pyramid, $2 \mathrm{kHz}$, normal incidence. (c) BEM prediction, square-based pyramid, $2 \mathrm{kHz}$, normal incidence. ments were mostly carried out at 1:5 scale. To avoid confusion, frequencies and distances given here are always equivalent full-scale values.

Unless otherwise stated, for hemispherical measurement the source was $15 \mathrm{~m}$ and the receivers $7.5 \mathrm{~m}$ from the surface, and for the single-plane measurement the source was 10 $\mathrm{m}$ and the receivers $5 \mathrm{~m}$ from the surface. One of the criteria given above for the diffusion coefficient is that it should be easy to measure. This is certainly true for the single-plane measurements, which are routinely undertaken on a boundary in a large room, which forms a pseudo-hemianechoic space, or an anechoic chamber. Measurements in the goniometer, however, require considerably more complex engineering to achieve the necessary microphone and source positioning in an anechoic chamber. They are also more time consuming due to the great increase in the number of measurements required. A spatial resolution of $5 \mathrm{deg}$ was used between receivers, resulting in 1369 measurement positions for a single angle of incidence. Figure 2 compares the measured and predicted scattering from a surface measured both on a single plane and a hemisphere. The agreement between theory and measurement is good. Incidentally, the measurement resolution of $5 \mathrm{deg}$ was chosen because tests showed that this was a sufficient resolution to gain the diffusion coefficient accurately without overburdening measurements with excessive sampling points.

Table I and Fig. 3 show some of the surfaces used in the test. The surfaces were chosen so that there was as little as possible redundancy in the surface set. In addition, each surface was chosen to test a particular attribute such as redirection, focusing, periodicity, randomness, partial absorption, good and poor diffusion. The concept was to cover a wide range of generic surface types to make the outcomes from the work as generalizable as possible. Incidentally, experience has shown that where possible the sample tested

TABLE I. Details of a selection of the samples measured.

\begin{tabular}{|c|c|c|}
\hline Sample & $\begin{array}{c}\text { Dimensions } \\
\text { (unless stated otherwise) }\end{array}$ & Reason for use \\
\hline Plane & $\begin{array}{l}\text { Various. } 0.57 \mathrm{~m} \text { wide for single } \\
\text { plane measurements }\end{array}$ & Reference. \\
\hline Concave prism & $1.5 \mathrm{~m}$ wide. & Practical worst-case diffuser. \\
\hline Cone & $1.5 \mathrm{~m}$ diameter, $0.35 \mathrm{~m}$ deep. & Redirects specular reflection. \\
\hline Square-based pyramid & $1.5 \mathrm{~m}$ square, $0.35 \mathrm{~m}$ deep. & Comparison with cone in $3 \mathrm{D}$. \\
\hline Periodic and random binary & $\begin{array}{l}\approx 3 \mathrm{~m} \text { square. Cross section of } \\
\text { parallel battens } 90 \mathrm{~mm} \text { square. }\end{array}$ & $\begin{array}{l}\text { To examine periodic and } \\
\text { aperiodic structures. }\end{array}$ \\
\hline Semicylinder & $0.55 \mathrm{~m}$ diameter. & Very effective diffuser. \\
\hline Triangular prism & $\begin{array}{l}0.54 \mathrm{~m} \text { wide, } 0.27 \mathrm{~m} \text { deep. Cross } \\
\text { section is right-angled isosceles } \\
\text { triangle. }\end{array}$ & Redirects in single plane. \\
\hline $\mathrm{QRD}^{\circledR}$ & $\begin{array}{l}N=7, \text { Maximum well depth } \\
0.2 \mathrm{~m} . \text { Well width } 60 \mathrm{~mm} .\end{array}$ & $\begin{array}{l}\text { Commercial single-plane } \\
\text { diffuser. }\end{array}$ \\
\hline Skyline ${ }^{\circledR}$ & $\approx 0.55 \mathrm{~m}$ square $\times 0.225 \mathrm{~m}$ deep. & $\begin{array}{l}\text { Commercial hemispherical } \\
\text { diffuser. }\end{array}$ \\
\hline BAD $^{\mathrm{TM}}$ Panel & $0.6 \mathrm{~m}$ square. & $\begin{array}{l}\text { Diffusion from variation in } \\
\text { surface absorption. } \\
\text { Commercial product. }\end{array}$ \\
\hline Periodic hemispheres & $\begin{array}{l}\text { Hemispheres are } 0.375 \mathrm{~m} \\
\text { diameter. Arrangements of } 1,7 \text {, } \\
\text { and } 19 \text { used. }\end{array}$ & $\begin{array}{l}\text { To compare single } \\
\text { and periodic arrangements }\end{array}$ \\
\hline
\end{tabular}


a)

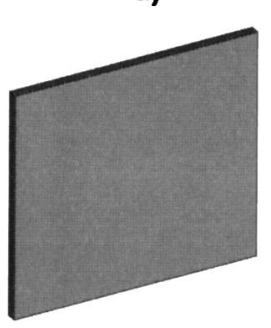

c)

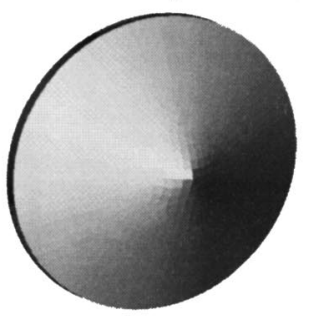

e)

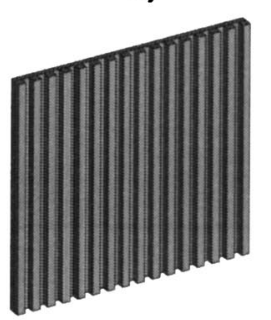

g)

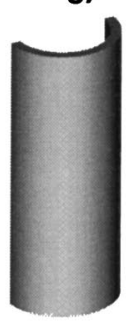

i)

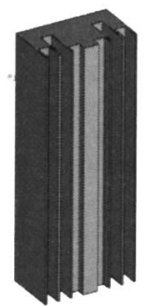

k)

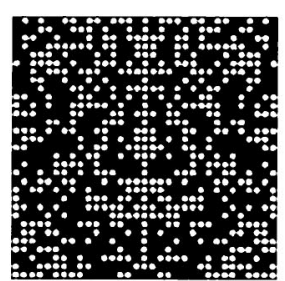

b)

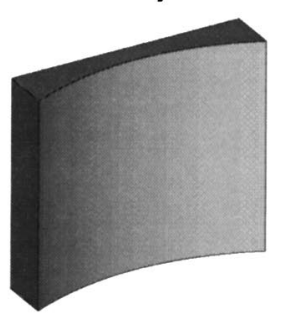

d)

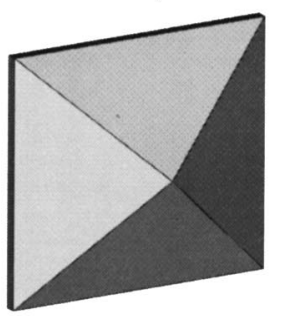

f)

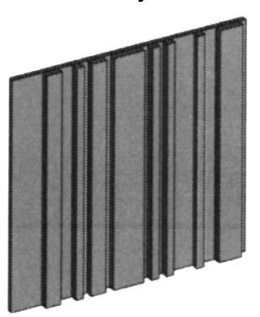

h)

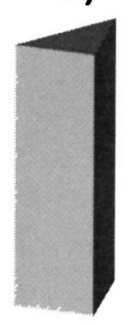

j)

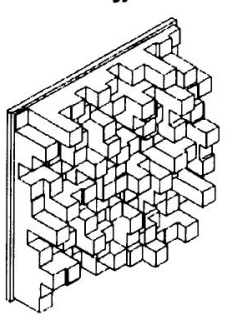

l)

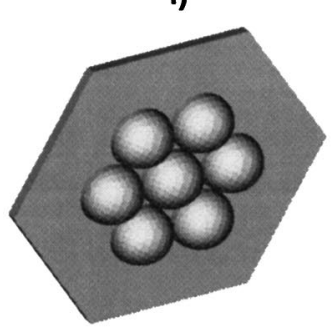

FIG. 3. Selection of samples measured. (a) Plane. (b) Concave prism. (c) Cone. (d) Square-based pyramid. (e) Periodic binary. (f) Random binary. (g) Semicylinder. (h) Triangular prism. (i) QRD $^{\circledR}$. (j) Skyline ${ }^{\circledR}$. (k) BAD ${ }^{\mathrm{TM}}$ Panel. (1) Periodic hemispheres.

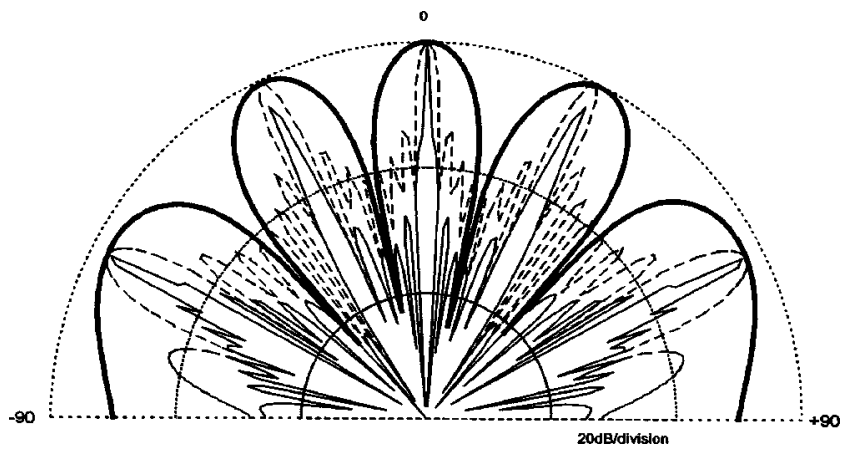

FIG. 4. Effect of number of periods of a diffuser on scattering lobe width. Single-plane Fraunhoffer prediction, $1130 \mathrm{~Hz}$, normal incidence. Quadratic residue diffuser, $N=53$, well width $12.7 \mathrm{~mm}$, design frequency $1130 \mathrm{~Hz}$. $-2,---8,-32$ periods.

should be the entire structure to be applied in a real application. This is because the method measures the diffraction effects of the edges and surface roughness together. While it may be possible to separate edge and surface diffraction effects for large surfaces with small roughness, for the majority of surfaces, such a separation is impossible. Where the whole sample cannot be tested, because of geometric constraints on source and receiver distance, the following techniques are suggested for reducing the sample size. For a periodic sample at least three complete repeat sequences should be included so the effects of lobing from repetition is measured. The width of the diffraction lobes, however, depends on the number of repeat units in the sample. ${ }^{18}$ This is illustrated in Fig. 4, where the scattering from a Schroeder diffuser is shown for 2, 8 , and 32 periods. Lobe narrowing is seen as the number of periods increases. So, if possible, the number of periods tested should be similar to the number used in real applications. The requirement for at least three periods is based on results from studies where an increasing number of periods of a sample were introduced and the effect on the diffusion coefficient monitored. For random surfaces, representative samples of the surface roughness should be tested, large enough so that surface rather than edge effects are more prominent in the scattering.

\section{NEAR AND FAR FIELDS}

Ideally, any diffusion coefficient should apply to all geometries that usually occur in room designs. One variable geometric factor is the distances from the source and receivers to the surface. All free-field measurements suffer from the problem that the relative levels within the polar response are dependent on these distances unless the source and receivers are in the far field. (The far field being where the scattered pressure falls by $6 \mathrm{~dB}$ per distance doubling for 3D geometries.) Unfortunately, in most room applications, it is usual for sources and receivers to be in the near rather than the far field. Figure 5 shows the scattering from a plane surface for a variety of receiver distances. As the receiver approaches the surface a top hat effect occurs; a plane surface appears to be a very good diffuser when measurements are close to the surface. (In fact, close enough to the surface, the reflection is provided by an approximate image source that radiates the same energy to all receivers except for any 


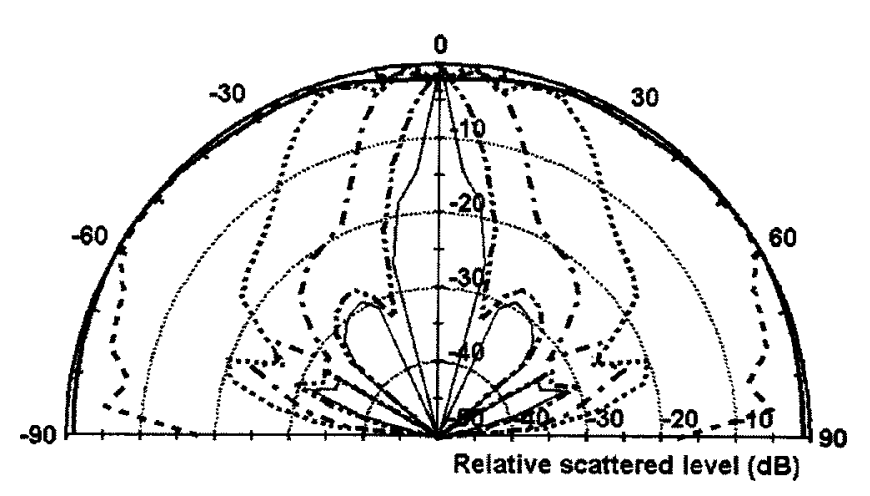

FIG. 5. Effect of receiver arc radius on the polar response of a $1 \mathrm{~m}$ square plane panel. Single-plane BEM prediction, $5 \mathrm{kHz}$, normal incidence, source distance $=100 \mathrm{~m} . \longrightarrow 0.1 \mathrm{~m},---0.5 \mathrm{~m},---1 \mathrm{~m},-\cdot-\cdot-\cdot-2 \mathrm{~m}$, -... - . $100 \mathrm{~m}$ m.

minor effects due to spherical spreading.) This seemingly contradicts conventional wisdom in room design, which would have us believe that a plane surface is a poor diffuser.

To understand this contradiction, it is necessary to understand why plane surfaces can cause problems in real applications. Problems can occur with plane surfaces with directional sources, such as trumpets. The reflected energy will be concentrated over a narrow solid angle, leading to a risk of detrimental effects such as echoes, coloration, or image shift. The results shown in Fig. 5 were produced using an omnidirectional source. Consequently, one solution would be to carry out the polar response measurements using a directional source that would better simulate the real sources that cause problems. But, the well-defined scientific nature and universality of the point-test source makes an omnidirectional source a better choice.

Another solution is to move to a different characterizing regime, ${ }^{9}$ as has been adopted for baffled surfaces, but this also has problems. Consequently, the preferred solution is for diffusion measurements based on polar distributions to be taken in the far field. There are standard formulations for approximately calculating the required distance for measurements to be in the far field. ${ }^{19}$ There are two criteria to satisfy: the receiver radius should be large compared to wavelength and the differences between path lengths from points on the surface to the receiver are small compared to wavelength. With the geometries and frequencies used for acoustic diffuser scattering, it is the latter criterion that is most exacting. Unfortunately, the common far-field formulations are not applicable to the case of oblique sources and receivers. In Fig. 6 the polar response from a 2-m wide plane surface is shown for four receiver radii. A distance correction of $1 / \sqrt{ } r$ has been used to correct for cylindrical wave spreading to aid comparison of the relative distributions. The first distance of 2.94 $\mathrm{m}$ represents the far-field criteria given in Ref. 18, and the distance of $12 \mathrm{~m}$ comes from a more complete consideration of Fresnel diffraction and the critical frequency for plane panels. ${ }^{20}$ Neither of these distances, however, is sufficient to get the true far-field response; this is achieved when the receiver radius is many hundreds of meters. Problems arise for angles where significant destructive interference occurs, because the amount of destructive interference is very sensitive to the relative magnitudes of the waves coming from the

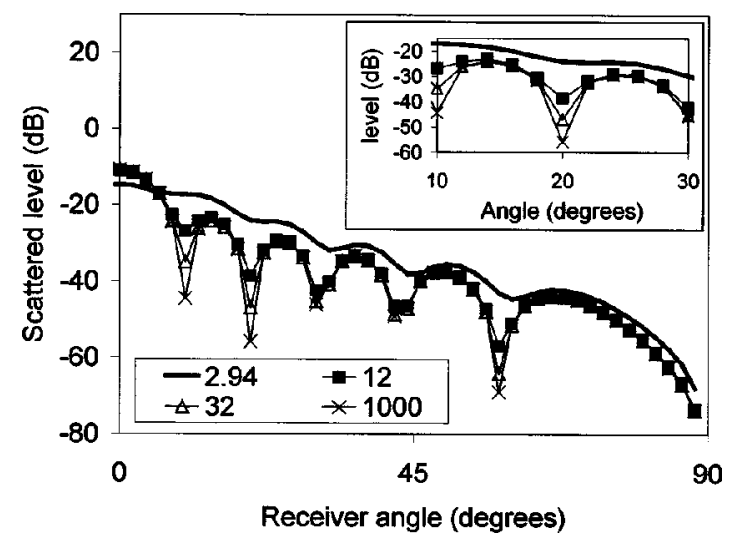

FIG. 6. Variation of scattered polar response with receiver distance, as shown in the legend, to illustrate extent of near field. Receiver angle on a linear scale for clarity; insert graph is an enlargement of a section of the main graph. $1 \mathrm{~m}$ plane surface at $1 \mathrm{kHz}$ using BEM predictions. A distance correction of $1 / \sqrt{ } r$ has been used to correct for cylindrical wave spreading.

secondary sources on the scattering surface (assuming the scattering is modeled following Huygen's principle). Consequently, the receiver distance required to achieve the true far field for oblique receivers is often so large that measurements cannot be accommodated in normal test facilities.

A pragmatic approach may be taken, however, to enable polar response measurements to be obtained using conventional methods and in normal test facilities. The calculation of the diffusion coefficient involves reducing the many scattered pressure values to a single figure of merit; consequently, detail such as the slight misrepresentation of the notches in the polar response will tend to average out. So, the true far field does not have to be obtained. It is sufficient to ensure that the majority of receivers is outside the specular zone so that the diffuser effects can be seen. Then, a reasonable approximation to the far-field diffusion coefficient value can be obtained. (The specular zone is defined in Fig. 7; it is the region over which a geometric reflection point exists on the surface. Although the specular zone is strictly a highfrequency construction, practice has shown it to be a useful concept for the geometries and frequencies typically used in diffuser design.) In the spatial domain, the effect of a diffuser should be to move energy from the specular zone to other positions. So, unless receivers are placed both outside and

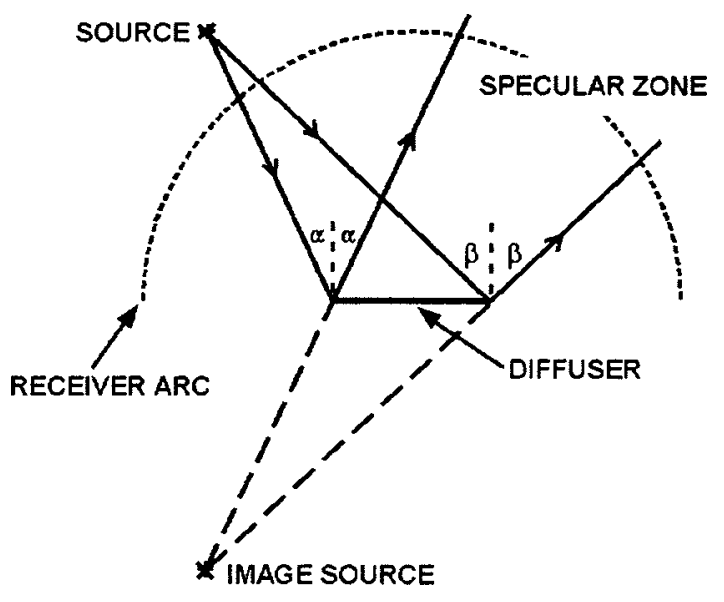

FIG. 7. Definition of specular zone. 


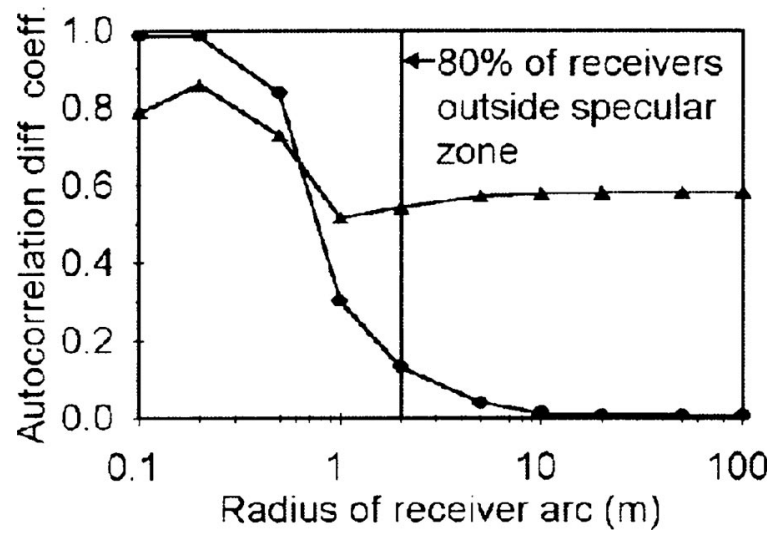

FIG. 8. Effect of receiver arc radius on a diffusion coefficient. Single-plane BEM predictions, normal incidence, source distance $=100 \mathrm{~m} .-1 \mathrm{~m}$ wide plane panel, $5 \mathrm{kHz}$. $-\mathbf{\Delta}-1 \mathrm{~m}$ wide random binary panel, $400 \mathrm{~Hz}$.

within the specular zone, measuring energy levels alone will not detect the effects of diffusion. In tests carried out for this project, typically $80 \%$ of receivers were outside the specular zone. In Fig. 8 the diffusion coefficient for two surfaces as a function of receiver distance is shown. The point where $80 \%$ of the receivers are outside the specular zone is shown. The plane panel case shown is one of the worst-case scenarios, and the error introduced into the diffusion coefficient is only 0.1 . Furthermore, this is a single frequency prediction. Once averaging effects across one-third octave bands are introduced, this error approximately halves. This illustrates that a reasonable approximation to the true far-field value can be obtained. Alternatively, systems such as near-field acoustic holography could be performed ${ }^{21}$ to enable near-field measurements to be projected into the far field, but they have their own different disadvantages such as the problems of mounting the surface in an application realistic manner. Another solution is to use validated prediction models; then, projecting to the far field is always possible.

For some surfaces, however, it is not sufficient just to measure in the far field. For concave surfaces, and others that might have significant aberrations closer to the surface, it is necessary to monitor in the near field as well as the far field to ensure that effects such as focusing are found. This is illustrated in Fig. 9, where the scattering from a concave surface is shown as a function of distance. It can be seen that receivers very close to the surface detect a good diffuser, but

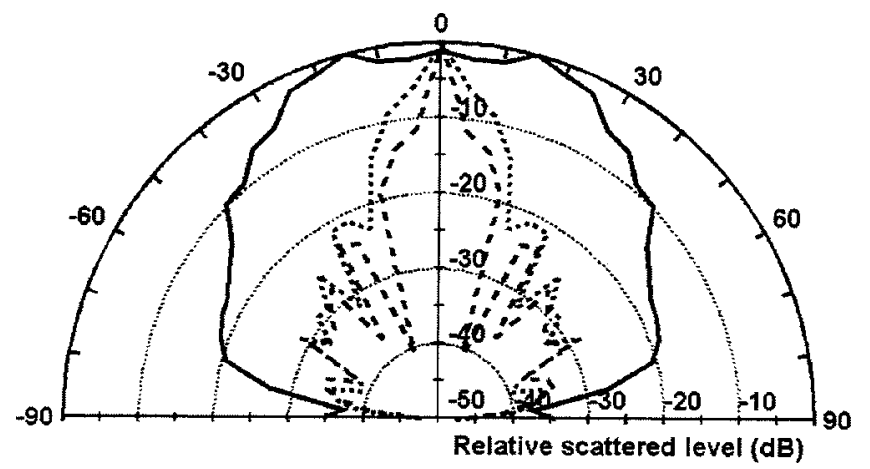

FIG. 9. Effect of receiver arc radius on the polar response of a concave prism. Single-plane BEM predictions, $2 \mathrm{kHz}$, normal incidence. Near field, --- focal distance, --- far field. a little further out the scattering becomes highly focused. This is as expected because the concave surface effectively focused the far-field scattered pressure distribution into the near field. In the far field, the diffusion is improved, the concave surface appearing to behave somewhat like a plane surface. In summary, a pragmatic approach requires receivers to be both inside and outside the specular zone; measurements at application realistic distances are also needed to check for focusing as a concave surface can focus far-field aberrations into the near field. If measurements are made at different radial distances from the surface, it is necessary to apply a correction to allow for the normal drop in level due to spherical or cylindrical spreading. Otherwise, the diffusion coefficient is overly biased by drops in levels that naturally occur due to effects that are not related to a surface's ability to diffuse. Incidentally, for the purpose of this paper, measures will be defined in terms of energy. Strictly speaking, as some of the measurements were not in the far field, the true outward propagating energy was not always measured. Consequently, in reality, measures are actually based on the squared pressure.

\section{SINGLE PLANE AND HEMISPHERICAL DIFFUSERS}

Diffusers can be designed to cause scattering in one or more planes. The examples of one-plane surfaces shown in Fig. 3 are the battens, cylinder, triangle, and one-dimensional quadratic residue diffuser (QRD). The terminology onedimensional arose from Schroeder-style diffusers, although it is less confusing to use the term single-plane diffusers. Often results for diffusion coefficients for single-plane surfaces are obtained from semicircular measurements in the plane of maximum diffusion. Strictly speaking, when evaluating the diffusion from such surfaces, it is best to calculate the diffusion coefficient in two directions - in the case of the cylinder across the width where diffusion is greatest and along the length where diffusion is smallest. More complex surfaces may create scattering in a more hemispherical manner. For example, an appropriate-sized sphere is effective at distributing energy in all directions. A two-dimensional Schroeder diffuser has two planes of maximum diffusion. In these cases, the polar distribution has to be measured over the surface of a hemisphere.

\section{REVIEW OF PREVIOUS COEFFICIENTS}

In this section, the previous parameters appearing in literature will be reviewed. The advantages and disadvantages of the coefficients will be presented, drawing on the large body of measured and predicted results generated for this work.

The simplest parameter to define is a measure that forms a ratio between the energy reflected outside the specular zone to the total reflected energy. This is similar in philosophy to the coefficient defined by Lam. ${ }^{22}$ If the squared pressure in a particular direction is given by $E(\Omega)$, then the coefficient, $d_{z}$, is given by 

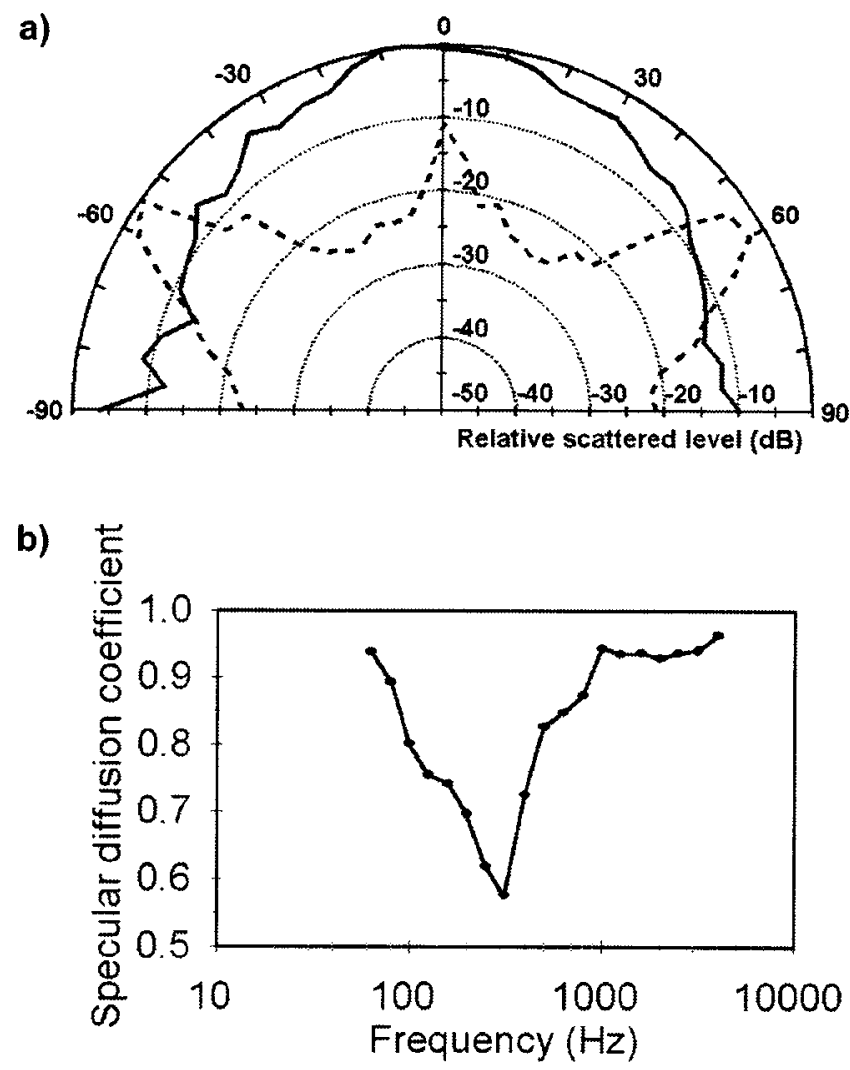

FIG. 10. Illustration of the simple specular zone diffusion coefficient failing due to incident sound being redirected as opposed to scattered. Two dimensional measurements of cone, normal incidence. (a) Polar responses: $250 \mathrm{~Hz}$, diffusion $=0.619$. $---4 \mathrm{kHz}$, diffusion $=0.963$. (b) Variation of the specular zone diffusion coefficient with frequency.

$$
d_{z}=1-\frac{\int_{s_{z}} E(\Omega) d \Omega}{\int_{s} E(\Omega) d \Omega}=1-\frac{\sum_{i=1,\left(\theta_{i}, \phi_{i}\right) \in s_{z}}^{n_{z}} E_{i} \Delta A_{i}}{\sum_{i=1}^{n} E_{i} \Delta A_{i}},
$$

where $s_{z}$ denotes the area of the specular zone; $s$ the area of a hemisphere or semicircle centered on the surface depending on whether this is a one-plane or hemispherical measure, and $\Omega$ is the solid angle. The second discrete form uses $n$ for the total number of measurements and $n_{z}$ the number of measurements in the specular zone. $\Delta A_{i}$ is the area sampled by the $i$ th measurement point. For a single-plane measurement with an evenly spaced set of receivers on an arc these factors can be ignored; for a hemispherical measurement, these give a weighting equivalent to changing the sampling to be linear with respect to area before calculation of the diffusion coefficient.

This definition has several qualities to recommend it. The definition is simple in concept, easy to explain, and links to some of the styles of diffusion modeling used in computer prediction models. Not only is it bounded, it also has values between the extremes which are easy to conceptualize. Furthermore, it links to the perceived role of diffusers in rooms, which is to move reflected energy from specular reflection directions.

There are, however, two main difficulties with the measure. The first is that according to Eq. (1) a complete diffuser would have no energy in the specular reflection direction and so creates a notch in the polar response. This is not a good definition of complete diffusion for two reasons. First, there are no known useful surfaces that can produce a significant notch in specular reflected energy over a significant bandwidth and for random incidence. While a primitive root diffuser and modified forms ${ }^{23-25}$ can generate notches at specific frequencies, the notches are not broadband. Using optimization $^{25}$ can produce a more broadband notch, but this can only be achieved for single specified angles of incidence. An alternative diffuser design, such as a simple triangle or pyramid, can produce such a notch, but this is achieved by redirection and not by dispersion. In addition, the notch is not achieved for all angles of incidence, and so these are not random incident surfaces. The second reason is that the term diffusion is defined as meaning to scatter in all directions, not to produce a specific diffusion distribution such as a notch. Consequently, it is a semantic misdefinition to term Eq. (1) a diffusion coefficient. Fortunately, a simple redefinition enables the above parameter to have complete diffusion as even scattering in all directions

$$
d_{z}^{\prime}=\left(1-\frac{\sum_{i=1, \theta_{i} \in s_{z}}^{n_{z}} E_{i} \Delta A_{i}}{\sum_{i=1}^{n} E_{i} \Delta A_{i}}\right) \frac{n}{n-n_{z}} .
$$

This does not cure the second difficulty with specular zone measures, which is the inability of the formulation to differentiate between redirection and dispersion. The scattering from a simple cone is shown as a function of frequency in Fig. 10 in terms of polar distributions and diffusion coefficients. The polar distributions show the scattering becoming distinctly less diffuse as frequency increases from 250 $\mathrm{Hz}$ to $4 \mathrm{kHz}$. At $250 \mathrm{~Hz}$ the cone is a reasonable diffuser; the surface roughness is small compared to wavelength and the cone behaves as a small flat surface, producing good diffusion because the surface size is much smaller than the wavelength. But, at $4 \mathrm{kHz}$ the surface detail of the cone sides is significant compared to wavelength and specular-like reflections off the two cone sides occur, producing two distinct lobes shown on the semicircular polar response. This is not seen in the calculated diffusion coefficient values, however, where the diffusion coefficient wrongly implies increasing diffuseness with frequency above $250 \mathrm{~Hz}$.

Diffuser designers, as discussed previously, have to worry about the difference between redirection and dispersion because specialist surfaces are used more often than not to treat first-order reflections. (Admittedly, specialist diffusers usually have some influence on the diffuseness of the reverberation in the space, but this is of secondary importance. Indeed, if the only consideration of diffuser design was to treat the diffuseness of the late-arriving sound field, surfaces which cause redirection would probably be just as effective as those that cause dispersion.) Consequently, specular zone measures in all their various guises are rejected.

The most popular form of diffusion definition, if this is measured on the number of publications using it, is the standard deviation. ${ }^{8-10}$ These have taken slightly different forms. 
Splitting these into generic types, two diffusion parameters, $D_{\sigma, E}$ and $D_{\sigma, L}$ can be defined

$$
\begin{aligned}
& D_{\sigma, E}=\sqrt{\frac{1}{n} \sum_{i=1}^{n}\left(E_{i}-\bar{E}\right)^{2}} ; \quad \bar{E}=\sum_{i=1}^{n} E_{i} \\
& D_{\sigma, L}=\sqrt{\frac{1}{n} \sum_{i=1}^{n}\left(L_{i}-\bar{L}\right)^{2}} ; \\
& \bar{L}=\sum_{i=1}^{n} L_{i} ; \quad L_{i}=10 \log _{10}\left(E_{i}\right) .
\end{aligned}
$$

These equations have assumed a single-plane measurement with evenly spaced receivers for clarity so the complication of terms to deal with uneven area sampling - seen in Eqs. (1) and (2) — can be removed. If all the energies in a polar distribution are the same, then the standard deviation is zero. Any deviation from the case of complete diffusion causes the standard deviation to increase. The parameters are simple in concept and relatively easy to explain as they use a common statistical operation. Values can be interpreted for average to good diffusers, as the standard deviation is a measure of spread, particularly if Eq. (4) is used. For example, two standard deviations would define within what limits $95 \%$ of scattered pressure levels lie if a normal distribution is assumed (incidentally, this is actually an incorrect supposition as most polar distributions are not normal distributions). For poor diffusers, as discussed below, the standard deviation formulation falls down and so does the usefulness of defining the range of levels for $95 \%$ of receivers.

The essential difference between Eqs. (3) and (4) is whether the standard deviation is taken of the measured levels or energy. The philosophy of taking the standard deviation of the levels is that the polar distribution of levels forms a more linear perceptual scale to listeners than the polar distribution of energies. Indeed, when evaluating the quality of diffusion, designers will view level polar distributions rather than those based on energy. So, the linear perceptual scale also applies to the visual evaluation of polar distributions used by practitioners. Unfortunately, the simple level formulation fails for poor diffusers. The highest standard deviation achievable occurs when all the scattered energy is reflected into half the receivers, the remaining receivers measuring the background level. (This occurs because level values are interval and not ratio.) This contradicts accepted philosophy of diffusers and reflectors where the worst case would be expected to be when all the scattered energy is reflected to one receiver. Consequently, although the standard deviation ranks moderate to good diffusers correctly, it does not rank poor diffusers in the right order. In fact, poor diffusers can be rated as very good. The problem is that the standard deviation measures the deviation from the mean. In Fig. 9, the scattering from a concave prism was shown at the focal distance. In this case the mean level is close to the low-level scattering, and so the standard deviation sees this as a reasonable diffuser as the vast majority of the scattering is close to the mean level. In reality, however, it is a very poor diffuser suffering from a strong specular reflection. A simple solution to this is to calculate the mean level via energy ${ }^{9}$

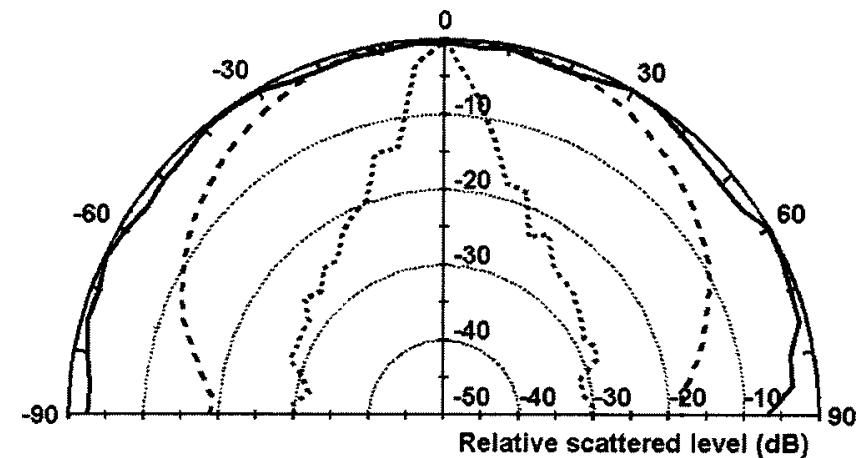

FIG. 11. Illustration of the ranking of polar responses by standard deviation. 2D measurements, normal incidence. - Convex semicylinder, $2 \mathrm{kHz}$, $d_{\sigma, E}=0.950$. --- Two concave semicylinders, $250 \mathrm{~Hz}, d_{\sigma, E}=0.748$. --Plane panel, $4 \mathrm{kHz}, d_{\sigma, E}=0.685$.

$$
\bar{L}=10 \log \sum_{i=1}^{n} 10^{L_{i} / 10}
$$

This then shifts the mean level upwards, so penalizing poor diffusers. It does not completely remove the problem. For example, when measuring a single-plane measurement over a semicircle with a $5^{\circ}$ resolution, the worst case occurs with all the reflected energy being scattered to 9 out of the 37 receivers. It has, however, ameliorated the problem to the point where it is more of a philosophical worry than a difficulty in real situations.

These types of standard deviation criteria have been used to design diffusers. ${ }^{8,9}$ Except for the difficulty discussed above, they have been shown to rank diffuser performance correctly. This is illustrated in Fig. 11. A further refinement of standard deviation coefficients was suggested by Angus; ${ }^{26}$ this was the concept of diffusion gain. He used this to evaluate the performance of large arrays of Schroeder diffusers. The diffusion gain is the ratio between the standard deviation values for the diffuser and an equivalent-sized flat surface. While this neatly illustrates the performance of diffusers over a flat surface, it is unbounded and so fails to meet a crucial criterion for a diffusion coefficient. In fact, the main problem with the standard deviation formulations is that they are not bounded in one direction. A normalization can be most readily achieved by considering the worse case. For Eq. (3), the worst case is where all the energy is scattered in one direction. Then, the diffusion coefficient, $d_{\sigma, E}$, is

$$
d_{\sigma, E} \approx 1-\frac{1}{n \bar{E}} \sqrt{\sum_{i=1}^{n}\left(E_{i}-\bar{E}\right)^{2}} ; \quad n \gg 1 .
$$

The subtraction from 1 is done so that the coefficient value is unity for a good diffuser and zero for a bad diffuser to be consistent with other measures given here. The diffusion coefficient now only depends on the relative distribution of energies within the polar response. It is independent of total scattered energy and hence any absorption that might occur. This seems a desirable characteristic, as there already exists a coefficient to measure absorption. For Eq. (4), the worst case is when all the scattered energy is evenly scattered into half the measurement points, and the other measurement points measure background noise. Using the mean of Eq. (5) results 


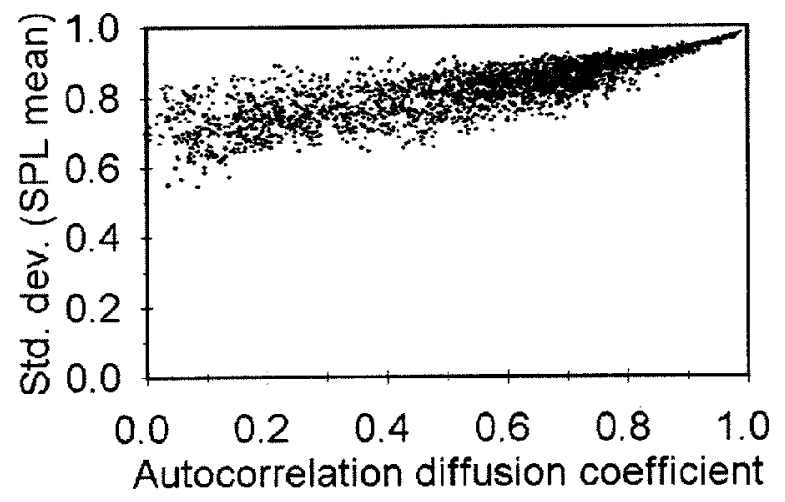

FIG. 12. Relationship between autocorrelation diffusion coefficient and standard deviation diffusion coefficient values for all 2D measurements and predictions. Each point represents a different measured frequency for a different surface.

in a different worst case that is $n$ dependent. Furthermore, once normalization has been performed, a problem arises because for many surfaces the diffusion coefficients are bunched at one end of the scale. This problem is even worse for the energy-based formulation than the level equation. In Fig. 12 the diffusion coefficients for a wide variety of surfaces is shown; it can be seen that really only about half the standard deviation scale is being used. For this reason, the standard deviation is no longer the favored parameter for characterizing scattering.

Another characterization method that has been suggested ${ }^{11,12}$ is based on a directivity-style measure. Subtle differences have appeared in the literature, but in the simplest, single-plane form, the coefficient, $d_{d}$, is

$$
d_{d}=1-\frac{1}{n} \sqrt{\sum_{i=1}^{n}\left(\frac{E_{i}}{\bar{E}}-1\right)^{2}} .
$$

Essentially, the directivity measures utilize the fact that complete diffusion occurs when the energy scattered in direction $i$ is a constant equal to the reciprocal of the number of measurements. While at first this appears to be a unique measure, it in fact is almost identical to the energy-based standard deviation normalized to between 0 and 1 as shown in Eq. (6) and so will not be discussed further.

Spherical harmonics have been suggested by Angus ${ }^{13}$ as another method for characterizing hemispherical scattering. The polar distributions are transformed into a set of amplitudes for the spherical harmonic basis functions. The fundamental spherical harmonic is a sphere and so represents even scattering in all directions. Higher harmonics are more complex in shape, for example dipoles, and so represent deviations from uniform scattering. A single-figure parameter can then be derived from the ratio of the fundamental to the higher-harmonics amplitudes. This coefficient has not been thoroughly tested and further work is needed to prove its worth. Conceptually, however, it has one major drawback. The calculation of the harmonics is complex, and difficult to explain to nonacademics. It is feared that this would make such a coefficient unacceptable to many practitioners.

In summary, all the published coefficients have flaws in one or more respects. Consequently, new coefficients have been developed to overcome some of the difficulties.
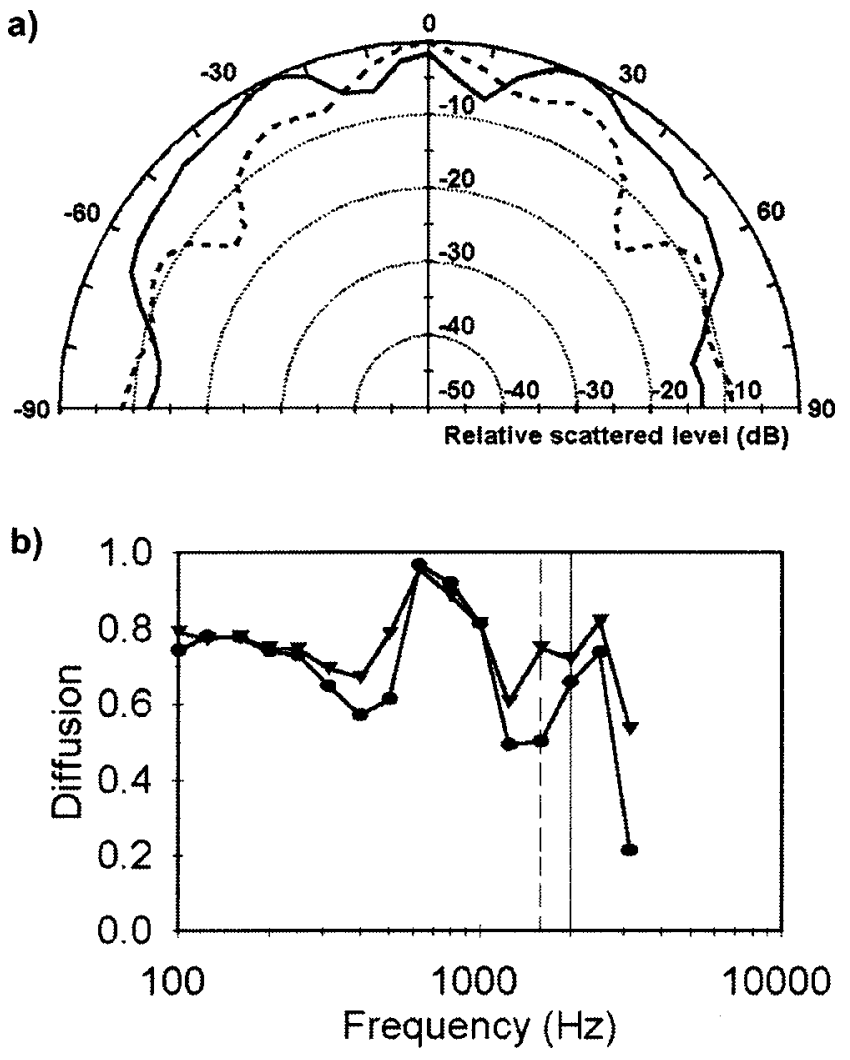

FIG. 13. Illustration of a case where $d_{90}$ fails to quantify diffusion correctly. 2D measurements of $N=7 \mathrm{QRD}$, normal incidence. (a) Polar responses: $-2 \mathrm{kHz},---1.6 \mathrm{kHz}$. (b) Variation of diffusion coefficient with frequency. - - Autocorrelation diffusion coefficient, $d_{a} ;-\boldsymbol{\nabla}-d_{90}$.

\section{NEW COEFFICIENTS}

A parameter can be defined in terms of the cumulative probability distribution. The advantage of doing this is that it creates a parameter where the physical meaning of all values can be easily interpreted. This $90 \%$ energy coefficient, $d_{90}$, is defined as follows:

$$
d_{90}=\frac{n_{90}}{0.9 n}
$$

where $n_{90}$ is the number of directions that $90 \%$ of the energy is scattered into and can be found from the cumulative probability distribution. The choice of $90 \%$ is purely empirical. Higher percentile values lead to a coefficient with a lack of discrimination and lower values lead to a coefficient which no longer ranks diffusers correctly. The coefficient can be easily interpreted. A value of 0.6 means that all the energy is scattered into $\approx 60 \%$ of the directions (strictly speaking, $54 \%)$. The coefficient works well for nearly all the required criteria; for example, it is bounded. The only problem is that for a small but significant minority of cases it fails to rank diffusers correctly. This is illustrated in Fig. 13. The solid line at $2 \mathrm{kHz}$ displays better diffusion than the dashed 1.6$\mathrm{kHz}$ line, yet the ranking shown by the $90 \%$ diffusion coefficient has it the wrong way around. Consequently, the following coefficient based on the autocorrelation function is preferred.

The autocorrelation function is most familiar in acoustics as a technique for assessing the similarity between two 


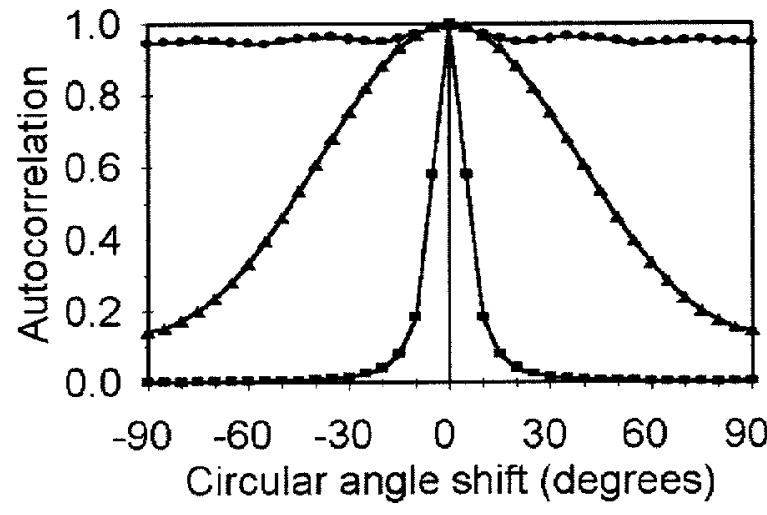

FIG. 14. Normalized circular autocorrelation functions of the polar responses in Fig. 9. - - Convex semicylinder, $2 \mathrm{kHz}$ - $-\mathbf{\Delta}-2 \mathrm{H}$ concave semicylinders, $250 \mathrm{~Hz}$. - $\mathbf{-}$ - Plane panel, $4 \mathrm{kHz}$.

or more sections of the same signal measured at different times. The function can also be used spatially to measure the similarity between different sections of polar responses. The technique is to first calculate the circular autocorrelation coefficient. Figures 11 and 14 show some typical polar responses and their circular autocorrelation functions. A perfect diffuser will have an autocorrelation value of 1 at all times; a complete specular reflector will only have a nonzero value at one sample point. The circular autocorrelation is then averaged across all displacements to give a single diffusion measure. At first this might appear to be a moderately involved calculation, but in fact the procedure reduces to a simple to calculate formulation. The autocorrelation diffusion coefficient, $d_{a}$, is given by

$$
d_{a}=\frac{\left(\sum_{i=1}^{n} E_{i}\right)^{2}}{n \sum_{i=1}^{n} E_{i}^{2}} .
$$

This is automatically bounded between $1 / n$ and 1 . A simple scaling can be carried out to make the bounding between 0 and 1 . The autocorrelation coefficient generally ranks diffusers in the same order as the energy-based standard deviation as there is a single-valued function relating the coefficients

$$
\frac{1}{d_{a}}=\left(1-d_{o, E}\right)^{2}(n-1)+1 .
$$

Essentially, the difference between standard deviation and autocorrelation is how the values are distributed along the diffusion axis. At this point, it would be good to know which scale, autocorrelation or standard deviation, forms the most linear scale in terms of the effect that diffusers have on the room acoustic and the response of listeners. But, this information is unavailable. Consequently, it is assumed that the autocorrelation is a better measure of diffusion than the standard deviation because practical values are distributed over the whole range of possible values rather than being bunched together. This is illustrated in Fig. 12. As the standard deviation has been thoroughly tested in diffuser design, this lends evidence that the autocorrelation ranks diffusers correctly. For example, in Fig. 11 the autocorrelation coefficients are $0.956,0.529$, and 0.055 for the semicylinder, two convex semicylinders, and plane surface, respectively. Further evidence of correct ranking can be seen in Fig. 15, where some

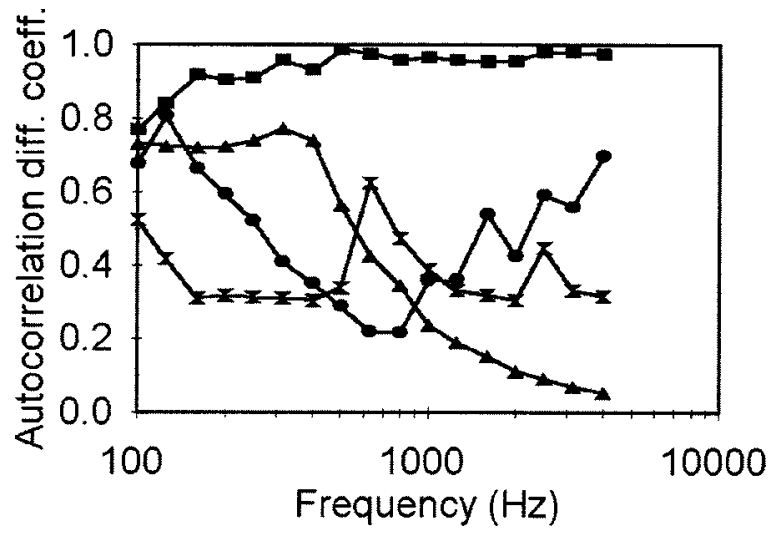

FIG. 15. Variation of $d_{a}$ with frequency for a selection of samples. 2D measurements, normal incidence. $-2 \times$ Skyline units differently orientated. $-\mathbf{\Delta}$ - Plane panel. $-\mathbf{-}-$ Convex semicylinder. $-\mathbf{X}-$ Random binary panel.

typical autocorrelation diffusion spectra are given. Again, the diffusion coefficient is independent of the absolute values and so the coefficient is correctly characterizing diffusion, not absorption. The only real drawback of the autocorrelation coefficient is that only the extremes of the scale are well defined. It is hard to put an exact physical meaning on intermediate coefficient values. Attempts were made to relate values to the spread of the polar distribution, but the relationship is too vague to be of much use and the necessary assumption of polar distribution normality is usually incorrect.

\section{CONCLUSIONS}

Techniques for characterizing the degree of diffusion produced by a surface have been discussed. This paper has concentrated on free-field methods. Previous suggested diffusion coefficients have been investigated and their strengths and weaknesses highlighted. There is no ideal diffusion coefficient that can meet all the desired criteria. For this reason, the new coefficient will not be perfect, but this is also true of the absorption coefficient that acousticians have been using for nearly a century. The best diffusion coefficient is the one that has fewest flaws and must be of use to practitioners. A new surface diffusion coefficient based on the autocorrelation function has been developed and demonstrated to be superior to previous measures.

\section{ACKNOWLEDGMENTS}

This work was funded by the Engineering and Physical Research Council (UK) (No. GR/L13124), with support from RPG Diffusor Systems, Inc.

${ }^{1}$ P. D'Antonio and T. J. Cox, "Diffusor application in rooms," Appl. Acoust. 60, 113-142 (2000).

${ }^{2}$ M. Vorländer, "International Round Robin on Room Acoustical Computer Simulations,' in Proceedings of the 15th ICA, Norway. II, pp. 689692 (1995).

${ }^{3}$ Y. W. Lam, "The dependence of diffusion parameters in a room acoustics prediction model on auditorium sizes and shapes,' J. Acoust. Soc. Am. 100, 2193-2203 (1996).

${ }^{4}$ Y. W. Lam, “A comparison of three diffuse reflection modeling methods 
used in room acoustics computer models," J. Acoust. Soc. Am. 100, 2181-2192 (1996).

${ }^{5}$ E. Mommertz and M. Vorländer, "Measurement of Scattering Coefficients of Surfaces in the Reverberation Chamber and in the Free Field," in Proceedings of the 15th ICA II (1995), pp. 577-580.

${ }^{6} \mathrm{E}$. Mommertz and M. Vorländer. The authors have submitted a paper on the method to a special edition of Applied Acoustics to be published in 1999.

${ }^{7}$ A. C. Pantelides, "The Effect of Absorbing and Diffusing Surfaces in a Rectangular Shaped Recording Studio," MSc thesis, University of Salford (1995).

${ }^{8}$ T. J. Cox, "Optimization of profiled diffusers," J. Acoust. Soc. Am. 97, 2928-2941 (1995).

${ }^{9}$ T. J. Cox, "Designing curved diffusers for performance spaces,' J. Audio Eng. Soc. 44, 354-364 (1996).

${ }^{10}$ P. D'Antonio, “The Disc Project: Directional Scattering Coefficient Determination and Auralization of Virtual Environments," in Proceedings Noise-Con 93 (May 1993), pp. 259-264.

${ }^{11}$ J. Angus, A. C. Marvin, J. Clegg, and J. F. Dawson, "A Practical Metric for Evaluating Sound Diffusers," Presented at the 98th Audio Engineering Society Convention, Preprint 3955 (D5) (February, 1995).

${ }^{12}$ D. Takahashi, "Development of optimum acoustic diffusers," J. Acoust. Soc. Am. 16, 51-58 (1995).

${ }^{13}$ J. A. S. Angus, "Diffuser assessment using surface spherical harmonics," J. Acoust. Soc. Am. 104, 1857-1858 (1998).

${ }^{14}$ T. J. Cox, "Predicting the scattering from reflectors and diffusers using 2D boundary element methods," J. Acoust. Soc. Am. 96, 874-878 (1994).

${ }^{15}$ T. J. Cox and Y. W. Lam, "Evaluation of methods for predicting the scattering from simple rigid panels," Appl. Acoust. 40, 123-140 (1993).
${ }^{16}$ T. J. Cox and Y. W. Lam, "Prediction and evaluation of the scattering from quadratic residue diffusers," J. Acoust. Soc. Am. 95, 297-305 (1994).

${ }^{17}$ P. D'Antonio, J. H. Konnert, and P. Kovitz, “The Disc Project: Experimental Measurement of the Directional Scattering Properties of Architectural Acoustic Surfaces,' 1p, AAd2, 141-144 (June 1994).

${ }^{18} \mathrm{P}$. D'Antonio and J. H. Konnert, "The reflection phase grating diffusor: Design theory and application," J. Audio Eng. Soc. 32, No.4, 228-238 (1984)

${ }^{19}$ L. E. Kinsler, A. R. Frey, A. B. Coppens, and J. V. Sanders, Fundamentals of Acoustics, 3rd ed. (Wiley, New York, 1982), pp. 187-188.

${ }^{20}$ J. H. Rindel, "Attenuation of Sound Reflections due to Diffraction," Nordic Acoustical Meeting. NAM86 (1986).

${ }^{21}$ M. Kleiner, H. Gustafsson, and J. Backman, "Measurement of directional scattering coefficients using near-field acoustic holography and spatial transformation of sound fields," J. Audio Eng. Soc. 45(5), 331-346 (1997).

${ }^{22}$ Y. W. Lam, "A boundary integral formulation for the prediction of acoustic scattering from periodic structures," J. Acoust. Soc. Am. 105(2), 762769 (1999).

${ }^{23}$ M. R. Schroeder, "Toward better acoustics for concert halls," Phys. Today 33(10), 24-30 (1980).

${ }^{24}$ E. Feldman, "A reflection grating that nullifies the specular reflection: A cone of silence,"' J. Acoust. Soc. Am. 98, 623-634 (1995).

${ }^{25}$ T. J. Cox, "Acoustic phase gratings for reduced specular reflection," Appl. Acoust. 60(2), 167-186 (2000).

${ }^{26}$ J. A. S. Angus, "Using Modulated Phase Reflection Gratings to Achieve Specific Diffusion Characteristics," presented at the 99th Audio Engineering Society Convention, Preprint 4117 (October 1995). 